\title{
Racial differences in the interaction between family history and risk factors associated with diabetes in the National Health and Nutritional Examination Survey, 1999-2004
}

\author{
Sunil Suchindran, ${ }^{1}$ Alicia M. Vana, MPH ${ }^{2}$, Richard A. Shaffer, PhD ${ }^{2}$, John E. Alcaraz, PhD', \\ and Jeanette J. McCarthy, PhD ${ }^{1,2}$
}

\begin{abstract}
Purpose: We sought to determine whether the association between family history, a surrogate for genetic predisposition, and diabetes was modified by any known diabetes risk factors and if these relationships were constant across different ethnic groups. Methods: We examined 10,899 adults from the National Health and Nutrition Examination Survey (1999-2004) to identify interactions between family history and clinical, demographic, and lifestyle variables for the outcome of diabetes using logistic regression analysis in racial/ethnic subgroups. Results: There was significant heterogeneity by race/ethnicity in the interaction between covariates and family history in relation to diabetes. In black $(P=0.0001)$ and Hispanic $(P=0.013)$, but not white $(P=0.75)$ subgroups, high-familial risk was a strong risk factor for diabetes among lean individuals but less so among overweight or obese subjects. Among blacks, high-familial risk conferred a 20 -fold increased odds of diabetes among lean subjects and only a sixfold increased odds among obese individuals. Conclusions: These findings suggest possible race/ ethnic-specific differences in gene by environment interaction and identify body mass index as an important effect modifier of familial risk in diabetes in non-white populations. These findings may help guide future genetic studies and improve the utility of family history as a public health screening tool. Genet Med 2009:11(7):542-547.
\end{abstract}

Key Words: family history, gene environment interaction, diabetes

A pproximately $7.0 \%$ of the US population have diabetes mellitus, and for the past 2 decades, the prevalence has been steadily increasing. ${ }^{1-3}$ Type 2 diabetes mellitus, which accounts for over $90 \%$ of all diagnosed cases, is a multifactorial disease with primary established risk factors including older age, physical inactivity, obesity, and family history of diabetes. Racial/ ethnic differences exist as well: diabetes rates among Hispanic subjects are 1.7-times higher and among blacks 1.8-times higher when compared to whites. ${ }^{1}$

Recent advances in genomic technologies have resulted in the identification of a number of new genetic risk factors yielding robust associations with type 2 diabetes. ${ }^{4-10}$ Despite their high level of statistical significance, most of the genetic variants

From the ${ }^{1}$ Center for Genomic Medicine, Institute for Genome Sciences and Policy, Duke University Medical Center, Durham, North Carolina; and ${ }^{2}$ Division of Epidemiology and Biostatistics, Graduate School of Public Health, San Diego State University, San Diego, California.

Jeanette McCarthy, PhD, Duke University Medical Center, 103 Research Drive, Box 3471, Durham, NC 27710. E-mail: jeanette.mccarthy@duke.edu

The authors declare no conflict of interest.

Submitted for publication June 19, 2008

Accepted for publication March 18, 2009.

Published online ahead of print April 25, 2009.

DOI: 10.1097/GIM.0b013e3181a70917 identified to date have weak effects (small odds ratios, ORs), making their clinical utility for predicting risk of diabetes less compelling. Interestingly, although the initial discoveries have been made primarily in white populations, the few published studies that have attempted to replicate these associations in non-white populations have in large part failed to do so. ${ }^{11-14}$ Nonetheless, full consideration has not been given to the possible modifying effect of important diabetes covariates on genetic risk factors, and whether ethnic differences in the distribution or effect of these risk factors would improve our ability to find genes underlying diabetes or enhance their generalizability or clinical utility once found.

In this study, we sought to identify covariates that broadly influence genetic susceptibility to diabetes, using family history as a surrogate, in the National Health and Nutrition Examination Surveys (NHANES) conducted from 1999 to 2004. Furthermore, we sought to determine whether there were racial/ethnic differences in these relationships that might explain some of the ethnic disparities in the prevalence of type 2 diabetes as well as the failure to replicate novel white genetic risk factors in black and Hispanic ethnic groups.

\section{METHODS}

\section{Study Population}

The NHANES is a sequential series of cross-sectional, nationally representative health examination surveys of the US civilian noninstitutionalized population. The surveys are conducted annually by the National Center for Health Statistics of the Centers for Disease Control and Prevention. Data for NHANES are from US households and are obtained using two methods: an in-home interview and a physical health examination. The institutional review board at the Centers for Disease Control and Prevention approved the study, and written informed consent was obtained from each participant. NHANES documentation and codebooks are available elsewhere. ${ }^{15}$

We merged three survey cycles to create an NHANES 19992004 data set $(N=31,126)$. The analyses were conducted using data for all participants who completed both the in-home interview and the physical health examination $(n=29,402)$. We excluded individuals who were $<30$ years of age at screening $(n=17,832)$, who developed diabetes before the age of $30(n=195)$, and who were pregnant $(n=903)$. Of the remaining 11,142 eligible persons, four were excluded from analysis because of missing or indeterminate self-reported diabetes status, and 239 were excluded for missing or indeterminate family history information. The final analytical sample consisted of 10,899 participants.

\section{Data Collection}

Our main outcome of interest, diabetes status, was based on self-report by asking whether a doctor or health professional 
had told the participant that he/she had diabetes or "sugar diabetes" other than during pregnancy (for female respondents). Measures of fasting glucose were available on a subset of the cohort and used in conjunction with self-reported family history as a confirmatory measure. For this definition, diabetes cases were those who either self-reported or had a fasting glucose measure of $\geq 126 \mathrm{mg} / \mathrm{dL}$ at enrollment. For family history, we assigned a stratified family history risk profile used by others and based on which relatives of a participant had diabetes. ${ }^{16-19}$ Individuals were classified as having a high-familial risk if at least two first-degree relatives had diabetes or if one first-degree relative and two second-degree relatives from the same lineage had diabetes. Individuals were classified as having a moderate familial risk if one first-degree relative had diabetes, and the number of second-degree relatives in the same lineage was less than two. Individuals were also assigned a moderate familial risk if no first-degree relatives had diabetes, but two second-degree relatives from the same lineage had diabetes. Individuals were classified as having an average familial risk if they did not have a family history of diabetes, if one second-degree relative had diabetes, or if the relative with diabetes was not a first-degree or a second-degree relative.

Several biologically relevant covariates were evaluated for their joint effect on diabetes outcome. Race/ethnicity was categorized as non-Hispanic white, non-Hispanic black, MexicanAmerican/Hispanic, and Other. Physical activity was categorized as ever/never based on questioning participants as to whether, over the past 30 days, they did any vigorous or moderate activities for at least 10 minutes. Vigorous activities were those that caused heavy sweating, or large increases in breathing or heart rate. Moderate activities were those that caused light sweating or a slight to moderate increase in breathing or heart rate. Hypertension was based on the interview and the medical exam. For the exam, blood pressure was measured three to four times. We discarded the first measurement and averaged the remaining measurements. We defined hypertension as having a diastolic pressure greater than or equal to 90 $\mathrm{mm} \mathrm{Hg}$, having a systolic pressure greater than or equal to 140 $\mathrm{mm} \mathrm{Hg}$, or indicating the use of a blood pressure medication.

High density lipoprotein (HDL) cholesterol and fasting triglycerides (collected on a random subset of individuals) were measured according to standard procedures ${ }^{15}$ and log transformed for analysis. Waist circumference was categorized $(<102 \mathrm{~cm}$ in men or $<88 \mathrm{~cm}$ in women) to define abdominal obesity. Body mass index (BMI, $\mathrm{kg} / \mathrm{m}^{2}$ ) was classified as normal (BMI $\left.<25\right)$, overweight (BMI between 25 and 29.9), and obese (BMI $\geq 30$ ). Dietary intake variables included saturated fatty acids ( $\mathrm{g}$ ) and total food energy (kcal), obtained from a 24-hour dietary recall. ${ }^{20}$

\section{Statistical Analysis}

All analyses were performed using the statistical package SAS, version 9.1 (SAS Institute Inc., Cary, NC). The ggplot2 package from the R statistical language was used to construct plots. ${ }^{21,22}$ To account for the complex, stratified, multistage probability cluster sampling design of NHANES, SAS Survey Procedures were used. These procedures incorporate statistical weights determined by the survey design. Exam weights were used if the analysis incorporated covariates obtained from the medical exam, and fasting weights were used for triglycerides, which is part of the fasting subsample. Four-year weights for the first two cycles were multiplied by $2 / 3$, and 2 -year weights from the last cycle were multiplied by $1 / 3$ so that the merged data represented an average year within the 6 years under study. To confirm results, we incorporated fasting glucose levels to model the prevalence of total diabetes. The subset with fasting glucose is considerably smaller. In addition, 403 individuals were excluded because they fasted $<8$ hours or $>23$ hours. Six-year fasting weights were formed in the same manner as 6-year exam weights. A combined weight was then used: 6-year exam weights were used for individuals with self-reported diabetes, and 6-year fasting weights were used for individuals with valid fasting glucose levels.

Comparisons between different groups were performed using $F$ test based on design-adjusted Rao-Scott $\chi^{2}$ for categorical variables and analysis of variance for continuous variables. Crude and adjusted odd ratios (ORs) and corresponding 95\% confidence intervals (CI) were calculated using logistic regression analysis. Potential confounders were determined by adding covariates individually to a logistic regression model and retaining those that changed the ORs by $10 \%$ or more. These included age, sex, education, income, insurance, access to care, physical activity, total energy intake, total saturated fatty acids, HDL cholesterol, triglycerides, abdominal obesity (waist), history of hypertension and BMI. Next, we examined interaction between family history and selected biologically relevant covariates including age, sex, physical activity, total energy intake, total saturated fatty acids, HDL cholesterol, triglycerides, abdominal obesity (waist), history of hypertension, and BMI. First, we tested the three-way interaction between family history, the covariate, and race. If the three-way interaction was significant, we conducted race-specific analyses and calculated ORs comparing family history at different levels of the covariate. If the three-way interaction was not significant, we dropped it from the model and constructed models to test two-way interactions between family history and the covariate for the entire analytical sample (races combined).

\section{RESULTS}

The estimated weighted prevalence of self-reported diabetes in the study population was $7.6 \%$ (95\% CI: $7.0-8.3)$. A highfamilial risk was found in $27 \%$ of diabetic subjects and $7 \%$ of nondiabetic subjects (Table 1), resulting in an unadjusted OR for having diabetes of 7.5 (95\% CI: 6.3-8.9) relative to an average familial risk. Moderate familial risk was found in $37 \%$ of diabetic subjects and $23 \%$ of nondiabetic subjects, yielding an unadjusted OR of 3.1 (95\% CI: 2.5-3.8) relative to having an average familial risk. Considering a range of diabetes covariates, only BMI and waist circumference were determined to be confounders of the relationship between family history and type 2 diabetes, as they each changed the OR for family history association with diabetes by $>10 \%$. We included only BMI as a confounder in the multiple regression models along with age, sex, and race. Addition of waist circumference did not change risk estimates further. The adjusted model resulted in only a slight attenuation of effect, producing ORs of 6.6 (95\% CI: 5.4-8.0) for the high-familial risk group and 3.1 (95\% CI: 2.5-3.8) for the moderate familial risk group. There were some differences in the magnitude of association between family history and diabetes by race (Table 2), with Hispanic subjects showing a stronger effect of family history than black or white subjects, but these differences were not statistically significant (family history by race interaction raw $P=0.11$ ).

To analyze the modifying effect of covariates on family history and examine race-specific effects, we began by testing three-way interactions between family history, race and each covariate (Table 3 ). We found significant three-way interactions between family history, race, and three different covariates: HDL cholesterol, triglycerides, and BMI (Bonferroni adjusted $P<0.05)$. Stratified by race, the familial risk by BMI interac- 
Table 1. Descriptive characteristics for demographic and risk factors of study participants with and without selfreported diabetes, adults aged 30 years and older, United States, 1999-2004

\begin{tabular}{|c|c|c|c|c|}
\hline Characteristic & Total $(N=10,899)$ & No diabetes $(n=9692)$ & Diabetes $(n=1207)$ & $P^{a}$ \\
\hline Age (yr) & $51.3(0.26)$ & $50.5(0.26)$ & $61.0(0.55)$ & $<0.0001$ \\
\hline HDL cholesterol $(\mathrm{mmol} / \mathrm{L})$ & $1.36(0.007)$ & $1.37(0.008)$ & $1.21(0.012)$ & $<0.0001$ \\
\hline Triglycerides $(\mathrm{mmol} / \mathrm{L})$ & $1.73(0.04)$ & $1.69(0.04)$ & $2.26(0.10)$ & $<0.0001$ \\
\hline Total energy intake (kcal/day) & $2145(14.1)$ & $2171(14.3)$ & $1833(33.6)$. & $<0.0001$ \\
\hline Total saturated fatty acids (g/day) & $26.6(0.21)$ & $26.8(0.22)$ & $23.1(0.62)$ & $<0.0001$ \\
\hline Sex & & & & 0.57 \\
\hline Male $(\%)$ & $5390(48)$ & $4787(48)$ & $603(49)$ & \\
\hline Female $(\%)$ & $5509(52)$ & $4905(52)$ & $604(51)$ & \\
\hline
\end{tabular}

Race/ethnicity

$\begin{array}{lc}\text { Non-Hispanic White (\%) } & 5653(74) \\ \text { Mexican-American/Hispanic (\%) } & 2784(11) \\ \text { Non-Hispanic Black (\%) } & 2120(10) \\ \text { Other (\%) } & 342(4)\end{array}$

$\begin{array}{cc}5184(75) & 469(65) \\ 2380(11) & 404(14) \\ 1824(10) & 296(16) \\ 304(4) & 38(5)\end{array}$

Physical activity

$\begin{array}{lc}\text { Ever (\%) } & 5669(60) \\ \text { Never (\%) } & 5227(40) \\ \text { BMI } & \\ \quad \text { Normal (\%) } & 3108(32) \\ \text { Overweight (\%) } & 3929(36) \\ \text { Obese (\%) } & 3459(32)\end{array}$

Abdominal obesity

Normal (\%) 4595 (47)

Obese $(\%)$

History of hypertension

Normal (\%)

Hypertension (\%)

Family history of diabetes

$\begin{array}{ll}\text { Average (\%) } & 6992(67) \\ \text { Moderate (\%) } & 2766(25) \\ \text { High (\%) } & 1141(8)\end{array}$

2919 (33.3)

3534 (36.3)

2906 (30.4)

4314 (49)

4896 (51)

$5272(65)$

3970 (35)

$6574(70)$

2328 (23)

790 (7)
$<0.0001$

189 (16)

395 (29)

553 (55)

$<0.0001$

281 (22)

834 (78)

$<0.0001$

$314(30)$

$856(70)$

$<0.0001$

$418(36)$

438 (37)

$351(27)$

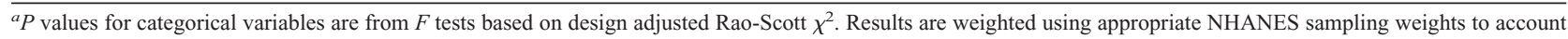
for unequal selection probabilities and nonrandom sampling design. Results are expressed as mean (SE) or number (\%).

tions were significant in both black (raw $P=0.0001$ ) and Hispanic (raw $P=0.013$ ) subpopulations. In these groups, the effect of family history was strongest in subjects with BMI in the normal range (Fig. 1). To test whether these results were driven by sibling effects alone, we constructed a variable representing sibling history and tested whether BMI modified its effect on diabetes. There was no evidence suggesting a strong sibling effect: $P=0.052$ in blacks and $P=0.63$ in Hispanics. In whites, the risk associated with familial history was constant across different BMI groups, with no evidence of interaction $(P=0.75)$. The significant three-way interactions with lipids were examined next. When stratified by race, the family historyHDL cholesterol and family history-triglyceride interactions were confined to the small group of subjects defined by "Other." Because this is a heterogeneous category including persons of multiracial, missing, or other racial background, these results were not considered further.

The above results for diagnosed diabetes are based on selfreport because incorporating fasting glucose to capture undiagnosed cases ( $3 \%$ of the population) resulted in a $50 \%$ reduction in sample size. Using fasting glucose to define total diabetesdiagnosed and undiagnosed diabetes - we observe an attenua- 
Table 2. Estimated adjusted and unadjusted odds ratios with $95 \%$ confidence intervals for the association between familial risk and self-reported diabetes in the NHANES population, 1999-2004, by race

\begin{tabular}{lcccc}
\hline & Unadjusted high-familial risk & Moderate familial risk & Adjusted $^{a}$ high-familial risk & Moderate familial risk \\
\hline All races & $7.5(6.3-8.9)$ & $3.1(2.5-3.8)$ & $6.6(5.4-8.0)$ & $3.1(2.5-3.8)$ \\
Whites & $7.4(5.8-9.5)$ & $2.9(2.2-3.8)$ & $7.0(5.3-9.0)$ & $2.9(2.1-3.8)$ \\
Hispanics & $9.0(6.4-12.6)$ & $4.1(2.9-5.8)$ & $8.4(5.6-12.7)$ & $4.1(3.0-5.8)$ \\
Blacks & $5.6(3.7-8.3)$ & $2.9(1.9-4.3)$ & $5.0(3.1-7.9)$ & $2.8(1.8-4.4)$ \\
\hline
\end{tabular}

${ }^{a}$ Adjusted for age, sex, and BMI. All odds ratios are relative to the average familial risk category.

Table 3. $P$ values for interaction between family history of diabetes and selected covariates with respect to selfreported diabetes in the NHANES population, 1999-2004

\begin{tabular}{|c|c|c|c|c|c|}
\hline & $P$, Three way & $P$, All & $P$, Hispanic & $P$, Black & $P$, White \\
\hline Age & 0.38 & 0.63 & - & - & - \\
\hline HDL cholesterol & 0.0001 & - & 0.10 & 0.49 & 0.13 \\
\hline Triglycerides & 0.0013 & - & 0.64 & 0.41 & 0.27 \\
\hline Total energy & 0.039 & 0.81 & - & - & - \\
\hline Fatty acids & 0.044 & 0.25 & - & - & - \\
\hline Sex & 0.19 & 0.67 & - & - & - \\
\hline Race/ethnicity & - & 0.11 & - & - & - \\
\hline Physical inactivity & 0.89 & 0.59 & - & - & - \\
\hline BMI & $<0.0001$ & - & 0.013 & 0.0001 & 0.75 \\
\hline Abdominal obesity & 0.086 & 0.15 & - & - & - \\
\hline Hypertension & 0.72 & 0.65 & - & - & - \\
\hline
\end{tabular}

tion of each OR, but the interaction remained borderline significant in blacks $(P=0.06)$, despite the much reduced sample size. In this subset, ORs for having a high-familial risk were as follows: in black subjects whose BMIs were defined as normal weight, overweight or obese, the ORs for the highest familial risk were 20.4 (95\% CI: 6.5-64.5), 5.2 (95\% CI: 2.2-12.3), and 5.0 (95\% CI: 2.5-10.3), respectively. Among Hispanics, the stratified ORs showed a similar trend to self-reported diabetes, but the interaction was no longer significant. ORs for the highest famlial risk in normal weight, overweight, and obese Hispanic subjects were 14.0 (95\% CI: 3.4-58.0), 5.6 (95\% CI: 1.8-17.3), and 8.5 (95\% CI: $3.8-19.4)$, respectively. The differences between ORs for diagnosed diabetes and total diabetes could result from an observed differential misclassification of diabetes between familial risk groups. We calculated the weighted percentage of individuals who reported that they are not diabetic but had fasting glucose values $>126 \mathrm{mg} / \mathrm{dL}$. These frequencies differed by familial risk groups, where we observed misclassifications rates of $2.6 \%, 4.1 \%$, and $7.2 \%$ for average, moderate, and high-familial risk groups, respectively.

\section{DISCUSSION}

In this study, we confirmed the strong relationship between family history and the prevalence of type 2 diabetes, consistent with numerous other studies, including a previous report in the NHANES population. ${ }^{19,23-36}$ However, we also found that the magnitude of association between family history and diabetes varied depending on the presence of other risk factors and that these relationships, in turn, varied by race/ethnicity. Among blacks in particular, a high-familial risk was a much stronger risk factor for diabetes among subjects with a normal BMI, increasing the odds approximately 20 -fold relative to subjects with an average family history. Similar, but less striking effects were seen in Hispanics, but among whites the modifying effect of BMI on familial risk was absent. Overall, our research suggests that the impact of family history is modified by BMI, and that this relationship varies significantly by race/ethnicity.

Ours is not the first study to explore interaction between family history and other risk factors for diabetes. However, few other studies examined risk factors besides obesity and sex, or racial/ethnic groups other than whites. There are numerous published studies addressing the interaction between obesity and family history on risk of type 2 diabetes, but the findings are inconsistent. ${ }^{29-32,37-43}$ Five studies (four among whites and one among Pima Indians) found that a combination of family history and increased body mass were most strongly associated with diabetes. ${ }^{30-32,37,39}$ However, only three of these formally tested interaction, resulting in interaction p-values between 0.01 and 0.05..$^{30,37,39}$ In contrast, five studies (three in white, one in 


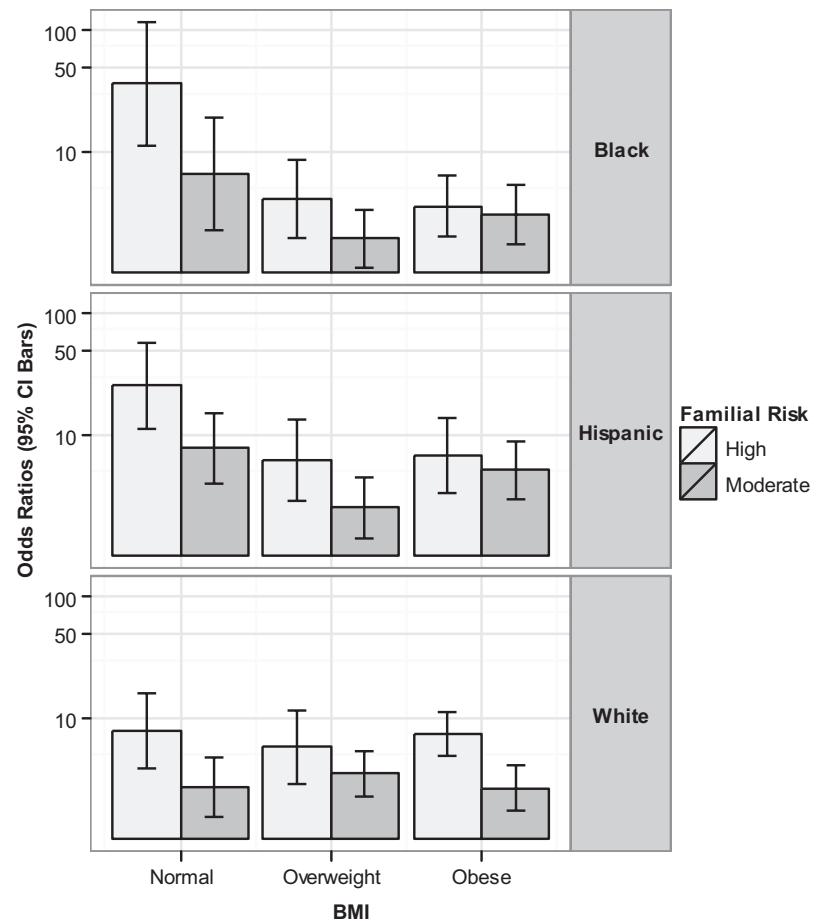

Fig. 1. Odds ratios (and 95\% confidence bars) for familial risk of self-reported diabetes for different categories of body mass index (BMI) in the three major race/ethnicity subgroups. Odds ratios compare high and moderate risks to the average familial risk and are adjusted for age and sex.

Japanese, and one in Pima Indian populations) reported that the combination of lean body mass and family history of diabetes conferred a greater risk of diabetes than having family history and a higher body mass, which agrees with our findings. But only two of these studies conducted a formal test of interaction: one in whites and one in Pima Indians. ${ }^{38,40-43}$ The study by Boer et al. ${ }^{44}$ in the elderly Dutch male cohort formally tested, but found no evidence of, interaction between obesity and family history, which is consistent with our finding of no effect in whites. To our knowledge, ours is the only published study to examine family history by obesity interaction among US Hispanic or black populations. In terms of other risk factors, we did not see a trend of a stronger relationship between family history and diabetes in men compared with women as noted in other studies, but it remains possible that three and four-way interactions that include sex are present. ${ }^{26,31,33}$

The main strength of this study is the use of a large, ethnically diverse population-based sample, and the formal analysis of interaction between covariates. Many of the other studies were carried out in individuals selected based on having diabetes or being overweight, introducing possible selection bias. Furthermore, a number of the studies failed to provide statistical support for reported interactions. One caveat of our study is the use of self-reported diabetes, which fails to take into account undiagnosed diabetes. We chose to use self-reported diabetes because inclusion of fasting glucose or other biometrics of diabetes reduced our sample size in half. Importantly, when we tested the association among the subset with fasting glucose, it showed that the resulting misclassification bias may have overestimated the effect sizes, so caution should be taken when interpreting the data based on self-reported diabetes status. Nonetheless, the interaction between BMI and family history in blacks remained significant.

Another drawback of using self-reported diabetes status is that knowledge of diabetes status could be influenced by family history of the disease and thus, differential misclassification of diabetes status is a possibility. Similarly, with respect to family history, previous studies have shown that persons with diabetes are more likely to recall a family member with diabetes than persons without the disease. ${ }^{45}$ In addition, both participant self-report of diabetes and family history variables did not differentiate between cases of type 1 and type 2 diabetes. However, we limited our analysis to subjects with age of onset of diabetes $>30$ years to minimize this bias.

The findings of our study have implications both for the design of genetic epidemiology studies and the use of family history as a risk predictor in clinical practice. If we believe family history to be a good surrogate of genetic risk, then studies aimed at discovering genes underlying type 2 diabetes would benefit from considering BMI levels of participants to enrich for subpopulations having a greater genetic predisposition. Specifically, in non-white populations, nonobese diabetic subjects may carry a higher genetic load than obese-diabetic subjects, and including this information may provide a useful strategy for finding genes of stronger effect. Similarly, once a gene is discovered, studies that determine the generalizability and utility of diabetes risk alleles should consider these important effect modifiers and race/ethnicity group differences in their effect. Of note, the few studies in African American, Hispanic, or Pima Indian populations that have tried to replicate previously identified diabetes risk alleles discovered in genome-wide association studies have failed to replicate many of these associations. ${ }^{11,13,14,46}$ Formal tests of genotype by obesity interaction or stratified association analysis based on obesity status would help to clarify this.

Finally, family history and other variables are used as risk predictors in clinical practice, but little consideration has been given to the interplay of family history with additional risk factors, nor to differences between race/ethnicity groups. Practically speaking, the results of our study suggest that perhaps in non-white populations, a high-familial risk of diabetes should be given much more weight in the absence of obesity. More research in this area is required to realize the potential clinical utility of both family history and newly discovered genetic markers of diabetes susceptibility.

\section{REFERENCES}

1. U.S. Department of Health and Human Services. National diabetes fact sheet: general information and national estimates on diabetes in the United States, 2005. Atlanta, GA: U.S. Department of Health and Human Services, Centers for Disease Control and Prevention, 2005.

2. Bishop D, BR Z, Roesler J. Diabetes. Chronic disease epidemiology and control, 2nd ed. Washington, DC: American Public Health Association, 1998.

3. Diagnosis and classification of diabetes mellitus. Diabetes Care 2007; 30(suppl 1):S42-S47.

4. Grant SF, Thorleifsson G, Reynisdottir I, et al. Variant of transcription factor 7-like 2 (TCF7L2) gene confers risk of type 2 diabetes. Nat Genet 2006; 38:320-323.

5. Sladek R, Rocheleau G, Rung J, et al. A genome-wide association study identifies novel risk loci for type 2 diabetes. Nature 2007:445:881-885.

6. Zeggini E, Weedon MN, Lindgren $\mathrm{CM}$, et al. Replication of genome-wide association signals in UK samples reveals risk loci for type 2 diabetes. Science 2007;316:1336-1341.

7. Scott LJ, Mohlke KL, Bonnycastle LL, et al. A genome-wide association study of type 2 diabetes in Finns detects multiple susceptibility variants. Science 2007;316:1341-1345.

8. Genome-wide association study of 14,000 cases of seven common diseases and 3,000 shared controls. Nature 2007;447:661-678. 
9. Saxena R, Voight BF, Lyssenko V, et al. Genome-wide association analysis identifies loci for type 2 diabetes and triglyceride levels. Science 2007;316: 1331-1336.

10. Steinthorsdottir V, Thorleifsson G, Reynisdottir I, et al. A variant in CDKAL1 influences insulin response and risk of type 2 diabetes. Nat Genet 2007;39:770-775

11. Palmer ND, Goodarzi MO, Langefeld CD, et al. Quantitative trait analysis of type 2 diabetes susceptibility loci identified from whole genome association studies in the Insulin Resistance Atherosclerosis Family Study. Diabetes 2008;57:1093-1100.

12. Lewis JP, Palmer ND, Hicks PJ, et al. Association analysis in african americans of European-derived type 2 diabetes single nucleotide polymorphisms from whole-genome association studies. Diabetes 2008;57: $2220-2225$.

13. Lehman DM, Hunt KJ, Leach RJ, et al. Haplotypes of transcription factor 7-like 2 (TCF7L2) gene and its upstream region are associated with type 2 diabetes and age of onset in Mexican Americans. Diabetes 2007;56: 389-393.

14. Guo T, Hanson RL, Traurig M, et al. TCF7L2 is not a major susceptibility gene for type 2 diabetes in Pima Indians: analysis of 3,501 individuals. Diabetes 2007;56:3082-3088.

15. Centers for Disease Control and Prevention (CDC). National Center for Health Statistics (NCHS). National Health and Nutrition Examination Survey. Available at: http://www.cdc.gov/nchs/nhanes.htm. Accessed November 2006.

16. Hariri S, Yoon PW, Moonesinghe R, Valdez R, Khoury MJ. Evaluation of family history as a risk factor and screening tool for detecting undiagnosed diabetes in a nationally representative survey population. Genet Med 2006; 8:752-759.

17. Hariri S, Yoon PW, Qureshi N, Valdez R, Scheuner MT, Khoury MJ. Family history of type 2 diabetes: a population-based screening tool for prevention? Genet Med 2006;8:102-108.

18. Scheuner MT, Wang SJ, Raffel LJ, Larabell SK, Rotter JI. Family history: a comprehensive genetic risk assessment method for the chronic conditions of adulthood. Am J Med Genet 1997;71:315-324.

19. Valdez R, Yoon PW, Liu T, Khoury MJ. Family history and prevalence of diabetes in the U.S. population: the 6-year results from the National Health and Nutrition Examination Survey (1999-2004). Diabetes Care 2007;30: 2517-2522.

20. Briefel RR, McDowell MA, Alaimo K, et al. Total energy intake of the US population: the third National Health and Nutrition Examination Survey, 1988-1991. Am J Clin Nutr 1995;62(suppl 5):1072S-1080S.

21. Wickham H. ggplot2: An implementation of the grammar of graphics. R package, 2008

22. Team RDC. R: a language and environment for statistical computing, 2008.

23. Meigs JB, Cupples LA, Wilson PW. Parental transmission of type 2 diabetes: the Framingham Offspring Study. Diabetes 2000;49:2201-2207.

24. Annis AM, Caulder MS, Cook ML, Duquette D. Family history, diabetes, and other demographic and risk factors among participants of the National Health and Nutrition Examination Survey 1999-2002. Prev Chronic Dis 2005;2:A19.

25. Schumacher MC, Hunt SC, Williams RR. Interactions between diabetes and family history of coronary heart disease and other risk factors for coronary heart disease among adults with diabetes in Utah. Epidemiology 1990;1:298-304.

26. Mitchell BD, Valdez R, Hazuda HP, Haffner SM, Monterrosa A, Stern MP Differences in the prevalence of diabetes and impaired glucose tolerance according to maternal or paternal history of diabetes. Diabetes Care 1993; 16:1262-1267.

27. Burchfiel CM, Curb JD, Rodriguez BL, et al. Incidence and predictors of diabetes in Japanese-American men. The Honolulu Heart Program. Ann Epidemiol 1990;5:33-43

28. Shaten BJ, Smith GD, Kuller LH, Neaton JD. Risk factors for the develop- ment of type II diabetes among men enrolled in the usual care group of the Multiple Risk Factor Intervention Trial. Diabetes Care 1993;16:1331-1339.

29. Knowler WC, Pettitt DJ, Savage PJ, Bennett PH. Diabetes incidence in Pima indians: contributions of obesity and parental diabetes. Am J Epidemiol 1981;113:144-156

30. Sargeant LA, Wareham NJ, Khaw KT. Family history of diabetes identifies a group at increased risk for the metabolic consequences of obesity and physical inactivity in EPIC-Norfolk: a population-based study. The European Prospective Investigation into Cancer. Int J Obes Relat Metab Disord 2000;24:1333-1339.

31. Gikas A, Sotiropoulos A, Panagiotakos D, Peppas T, Skliros E, Pappas S. Prevalence, and associated risk factors, of self-reported diabetes mellitus in a sample of adult urban population in Greece: MEDICAL Exit Poll Research in Salamis (MEDICAL EXPRESS 2002). BMC Public Health 2004;4:2.

32. Grill V, Persson G, Carlsson S, et al. Family history of diabetes in middleaged Swedish men is a gender unrelated factor which associates with insulinopenia in newly diagnosed diabetic subjects. Diabetologia 1999;42: $15-23$

33. Kuhl J, Hilding A, Ostenson CG, Grill V, Efendic S, Bavenholm P. Characterisation of subjects with early abnormalities of glucose tolerance in the Stockholm Diabetes Prevention Programme: the impact of sex and type 2 diabetes heredity. Diabetologia 2005;48:35-40.

34. Bjornholt JV, Erikssen G, Liestol K, Jervell J, Thaulow E, Erikssen J Type 2 diabetes and maternal family history: an impact beyond slow glucose removal rate and fasting hyperglycemia in low-risk individuals? Results from 22.5 years of follow-up of healthy nondiabetic men. Diabetes Care 2000;23:1255-1259.

35. Thorand B, Liese AD, Metzger MH, Reitmeir P, Schneider A, Lowel H. Can inaccuracy of reported parental history of diabetes explain the maternal transmission hypothesis for diabetes? Int J Epidemiol 2001;30:1084-1089.

36. Erasmus RT, Blanco Blanco E, Okesina AB, Mesa Arana J, Gqweta Z, Matsha T. Importance of family history in type 2 black South African diabetic patients. Postgrad Med J 2001;77:323-325.

37. Morris RD, Rimm AA. Association of waist to hip ratio and family history with the prevalence of NIDDM among 25,272 adult, white females. Am J Public Health 1991;81:507-509.

38. Hanson RL, Pettitt DJ, Bennett PH, et al. Familial relationships between obesity and NIDDM. Diabetes 1995;44:418-422.

39. van Dam RM, Boer JM, Feskens EJ, Seidell JC. Parental history of diabetes modifies the association between abdominal adiposity and hyperglycemia. Diabetes Care 2001;24:1454-1459.

40. Lemieux S, Despres JP, Nadeau A, Prud'homme D, Tremblay A, Bouchard C. Heterogeneous glycaemic and insulinaemic responses to oral glucose in non-diabetic men: interactions between duration of obesity, body fat distribution and family history of diabetes mellitus. Diabetologia 1992;35:653-659.

41. Kobberling J. Studies on the genetic heterogeneity of diabetes mellitus. Diabetologia 1971;7:46-49.

42. Baird JD. Diabetes mellitus and obesity. Proc Nutr Soc 1973;32:199-203.

43. Kuzuya T, Matsuda A. Family histories of diabetes among Japanese patients with type 1 (insulin-dependent) and type 2 (non-insulin-dependent) diabetes. Diabetologia 1982;22:372-374.

44. Boer JM, Feskens EJ, Kromhout D. Characteristics of non-insulin-dependent diabetes mellitus in elderly men: effect modification by family history. Int $J$ Epidemiol 1996;25:394-402.

45. Awareness of family health history as a risk factor for disease. Morbid Mortal Week Rep 2004;53:1044-1047.

46. Lewis JP, Palmer ND, Hicks PJ, et al. Association Analysis of EuropeanDerived Type 2 Diabetes SNPs from Whole Genome Association Studies in African Americans. Diabetes 2008;57:2220-2225. 